\title{
Morfologia e reprodução de Chondria curvilineata F.S. Collins \& Hervey (Rhodomelaceae, Rhodophyta), uma adição à flora brasileira
}

\author{
Martha Böker-Torres ${ }^{1}$, Paulo Antunes Horta ${ }^{1,4}$, José Salles, Luciane Ouriques ${ }^{2}$, Mutue Toyota Fujii ${ }^{3}$ e \\ Zenilda Bouzon ${ }^{2}$
}

Recebido em 6/08/2007. Aceito em 25/04/2008

\begin{abstract}
RESUMO - (Morfologia e reprodução de Chondria curvilineata F.S. Collins \& Hervey (Rhodomelaceae, Rhodophyta), uma adição à flora brasileira). O trabalho a espécie Chondria curvilineata é escrita pela primeira vez para o litoral brasileiro a. O material foi coletado no sublitoral da Lagoa da Conceição, Florianópolis, Santa Catarina. É apresentada uma descrição detalhada da morfologia e reprodução, discutindo e comparando as características diagnósticas da referida espécie com as demais espécies descritas para o Brasil e para outras partes do mundo.
\end{abstract}

Palavras-chave: Chondria, Lagoa da Conceição, macroalgas, Santa Catarina, taxonomia

\begin{abstract}
Morphology and reproduction of Chondria curvilineata F.S. Collins \& Hervey (Rhodomelaceae, Rhodophyta), an addition to the Brazilian marine flora). Here the species Chondria curvilineata is described for the first time to the Brazilian coast. The material was collected in the subtidal region of Lagoa da Conceição, Florianópolis, Santa Catarina. We present a detailed description of the morphology and reproduction, discussing and comparing the diagnostic characteristic.
\end{abstract}

Key words: Chondria, Lagoa da Conceição, Santa Catarina, seaweed, taxonomy

\section{Introdução}

O gênero Chondria foi proposto por C. Agardh (1817) e está representado atualmente por 75 espécies distribuídas em regiões tropicais e temperadas quentes de todo o mundo (Tani et al. 2003; Tani \& Masuda 2003). Para o Brasil são listadas, até o momento, nove espécies, sendo estas referidas principalmente para o sudeste do país (Joly 1957; 1965; Oliveira Filho 1969; Ugadim 1976; Bacci 2005). Para o estado de Santa Catarina é citada apenas a espécie Chondria polyrhiza Collins \& Hervey (Cordeiro-Marino 1978).

A morfologia das espécies de Chondria é bastante variada, tanto na altura, hábito e nos detalhes anatômicos, mas as principais características distintivas dos demais gêneros de Rhodomelaceae é a presença de talo corticado, polissifônico e constituído por cinco células pericentrais grandes e com célula apical sempre visível, mesmo naquelas com depressão apical. Durante os estudos das macroalgas do litoral de Santa Catarina, uma espécie com as características morfológicas de Chondria curvilineata F.S. Collins \& Hervey foi encontrada. As principais características diagnósticas que suportam esta identificação são: ápice dos últimos râmulos truncados e com paredes distais das células apicais conspicuamente espessadas (Wynne 1993).

A espécie foi originalmente descrita para Bermuda (Collins \& Hervey 1917) e posteriormente para outras regiões caraíbicas (Taylor 1960; Littler \& Littler 2000; Cabrera et al. 2004), mas a sua distribuição é ampla, com registro na costa nordeste da América do Norte (Schneider \& Searles 1991), na Europa (Audiffred \& Weisscher 1984; Price et al. 1986; John et al. 2004), no Mediterrâneo (Gómez Garreta et al. 2001, Cormaci et al. 2004), leste da África e Índia (Silva et al. 1996; Lipkin \& Silva 2002), sudeste da Ásia (Silva et al. 1987), Ilhas Atlânticas (Taylor 1960; Schneider \& Searles 1991), Carolina do Norte, EUA (Schneider \& Searles 1991) e Filipinas (Meñez \& Calumpong 1981).

Este trabalho tem por objetivo registrar pela primeira vez para o litoral brasileiro Chondria curvilineata e descrever a morfoanatomia de aspectos vegetativos e reprodutivos da referida espécie.

\footnotetext{
1 Universidade Federal de Santa Catarina, Departamento de Botânica, 88010-970 Florianópolis, SC, Brasil

2 Universidade Federal de Santa Catarina, Departamento de Biologia Celular e Genética, 88010-970 Florianópolis, SC, Brasil

3 Instituto de Botânica, Seção de Ficologia, C. Postal 3005, 01061-970 São Paulo, SP, Brasil

4 Autor para correspondência: pahorta@ccb.ufsc.br.
} 


\section{Material e métodos}

Os espécimes foram coletados no canal que liga a Lagoa da Conceição $\left(27^{\circ} 35^{\prime} 19^{\prime \prime}\right.$ 'S 48²6'10"W) com o mar com a utilização de mergulho livre de 1 a 2 metros de profundidade (Fig. 1) em 4 expedições: 8/04/2002, 17/06/2002, 7/12/2002 e 7/09/2004. Após cada coleta, o material foi acondicionado em sacos plásticos devidamente etiquetados e levado para o laboratório, onde as amostras foram triadas, fixadas em formaldeído a $4 \%$ em água do mar. Para os estudos morfológicos e anatômicos de estruturas vegetativas e reprodutivas, foram feitos cortes histológicos com auxílio de lâmina de barbear, utilizando-se azul de anilina a 1\% (Wiseman 1976) para observação das estruturas de reprodução.

O material foi examinado em microscópios óptico Carl Zeizz Jena e estereoscópico Olympus SZ 40. A documentação fotográfica foi feita com câmera Olympus C-35 AB, acoplada ao microscópio Olympus BH-2 ou ao microscópio estereoscópico Olympus SZ-PT 11. O material identificado foi depositado no Herbário do Departamento de Botânica da Universidade Federal de Santa Catarina, Brasil (FLOR).

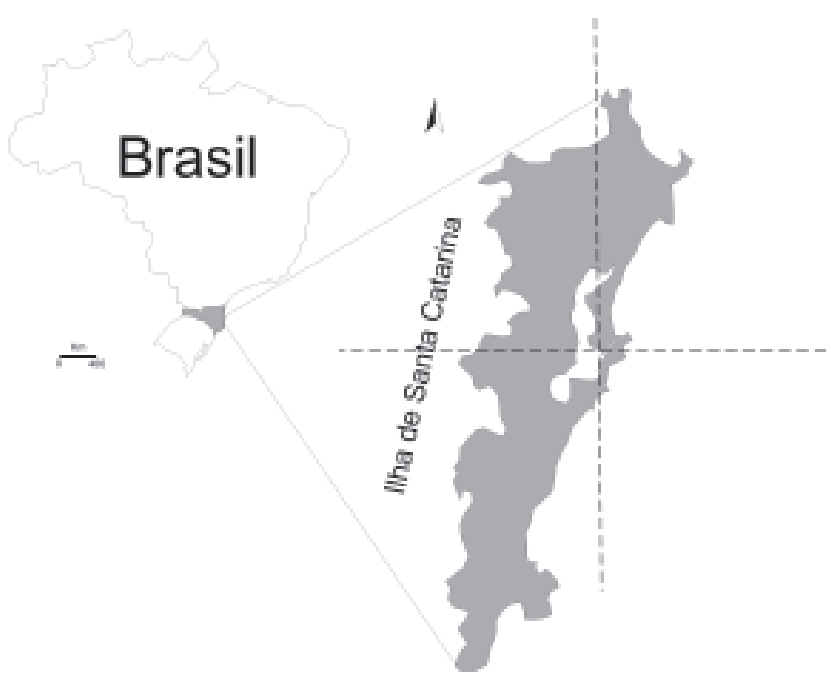

Figura 1. Mapa da localização da estação de coleta (seta) no Canal de Comunicação com o mar da Lagoa da Conceição, Florianópolis, SC, Brasil (27³5'19”'S; 48²6'10”'W).

Chondria curvilineata F.S. Collins \& Hervey, Proc. Amer. Acad. Arts \& Sci. 1817, p.

Localidade tipo: Bermuda.

Fig. 2-13

Alga com talo cilíndrico suavemente carnudo, marrom-avermelhada, de hábito prostrado na base e ereto acima, com até $3 \mathrm{~cm}$ compr. Fixação por meio de um disco primário medindo 180-508 $\mu \mathrm{m}$ e secundaria- mente por meio de rizóides, com 355,70-121,90 $\mu \mathrm{m}$ compr. e hápteros ao longo de todos os ramos decumbentes.

Eixo principal prostrado, com ramos secundários eretos. Ramos eretos medindo em torno de 330 a $700 \mu \mathrm{m}$ diâm., apresentando constrição próximo à base dos ramos laterais. Ramificação alterna, destacando-se por apresentar mais de um ramo nascendo de uma mesma região do talo. Diâmetro do eixo principal entre 330-700 $\mu \mathrm{m}$ na base e 300-600 $\mu \mathrm{m}$ na região mediana. Ápice obtuso, evidenciando o crescimento por uma célula apical, com tricoblastos longos e numerosos. Células corticais em vista superfícial alongadas, medindo 35-98 $\times 8-30 \mu \mathrm{m}$, com paredes nitidamente espessadas. Em corte transversal, cada célula axial produz cinco células pericentrais e uma a duas camadas de células corticais, com espaços intercelulares entre elas. Espessamentos lenticulares do tipo "cap" presentes. Em corte longitudinal do talo, paredes das células corticais não projetadas além da superfície do talo.

Gametófitos dióicos, com espécimes masculinos apresentando corpos espermatangiais desenvolvidos a partir da base dos tricoblastos modificados, nas porções apicais, com apenas uma camada de células estéreis radialmente alongadas, dando ao ramo aspecto de ápice ramificado. Quando maduros, os corpos espermatangiais organizam-se em estrutura de forma orbicular, pedunculado, medindo 88-338 $\mu \mathrm{m}$ diâm. Espécimes femininos apresentam carposporófitos medindo de 36-104 um diâm., com pericarpo desenvolvendo-se desde as fases iniciais da formação do cistocarpo. Cistocarpos ovóides, laterais, adanatos, dispostos adaxialmente nos ramos de última ordem, medindo ca. 800-1000 بm diâm.

Tetrasporófitos com 2-5 tetrasporângios por segmento axial, originados a partir de células pericentrais. Cada célula pericentral forma duas células préesporangiais, o próprio tetrasporângio na posição abaxial e, por último, a célula pós-esporangial, que contribuirá para o sistema de corticação do talo. Tetrasporângios quando maduros medem de 63-80 $\mu$ m diâm. e distribuem-se em porções distais dos eixos e em ramos laterais curtos e cilíndricos.

Material examinado: BRASIL. Santa Catarina: Canal da Barra da Lagoa (27³5'19"S; 48²6'10”W), gametófitos masculinos, gametófitos femininos e tetrasporófitos 20/VI/2002, Böker-Torres \& Horta 235 (FLOR 14360, 14400); Canal da Barra da Lagoa (27³5'19'S; 48²6'10'W), gametófitos masculinos e tetrasporófitos 18/IX/2002, Salles 380, 381 (FLOR 14505, 14506).

Comentários: todos os exemplares foram encontrados crescendo no sublitoral, de 1 a 2 metros de 
profundidade, em agregados de hábito epilítico ou sobre algas maiores como Acantophora spicifera (Vahl) Børgesen. Foram encontrados indivíduos férteis próximo à ponte que liga a Lagoa da Conceição ao Canal da Barra em todas as coletas, apresentando gametófito feminino e masculino durante o inverno e tetrasporófito em todas as coletas.

\section{Discussão}

Os aspectos morfológicos dos espécimes estudados no presente trabalho correspondem com as descrições originais de Chondria curvilineata (Collins \& Hervey 1917) e de Taylor (1960), Schneider \& Searles (1991) e Tani \& Masuda (2003), por apresentar hábito epifítico,

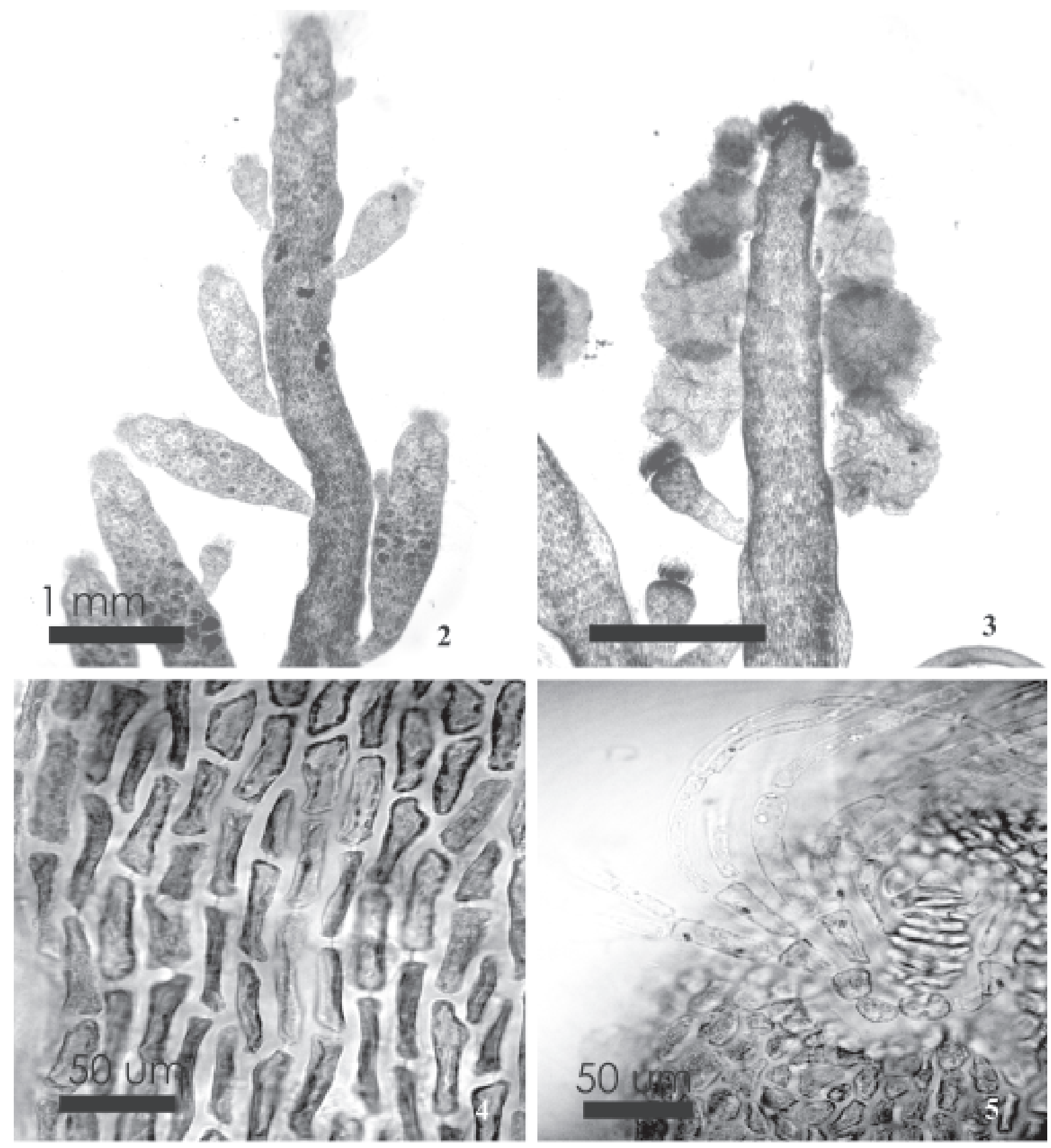

Figura 2-5. Chondria curvilineata Collins \& Hervey. 2. Aspecto Geral de planta tetrasporofítica evidenciando ramos laterais com constrição na base (setas). 3. Aspecto Geral de planta gametofítica masculina. 4. Vista superficial do talo evidenciando células corticais alongadas longitudinalmente. 5. Detalhe de um ramo lateral jovem com tricoblasto basal. 
natureza do eixo principal prostrada, fixação do talo por meio de um único apressório discóide e râmulos com constrição proximal. Em vista superficial do talo, as células corticais são estreitas e alongadas, mas não projetadas além da superfície do talo. O segmento axial apresenta cinco células pericentrais como nas demais espécies do gênero. Presença de espessamentos lenticulares nas paredes das células pericentrais também é característica da espécie.
Collins \& Hervey (1917) descreveram C. curvilineata com eixos medindo 200 a $500 \mu \mathrm{m}$, similares aos observados neste estudo. Entretanto, os espécimes aqui estudados diferem do material original de C. curvilineata e dos descritos por Schneider \& Searles (1991) por apresentar apenas uma camada de células corticais ao invés de uma a duas como nas anteriores.

Collins \& Hervey (1917) e Schneider \& Searles (1991) descreveram as células superficiais com medidas
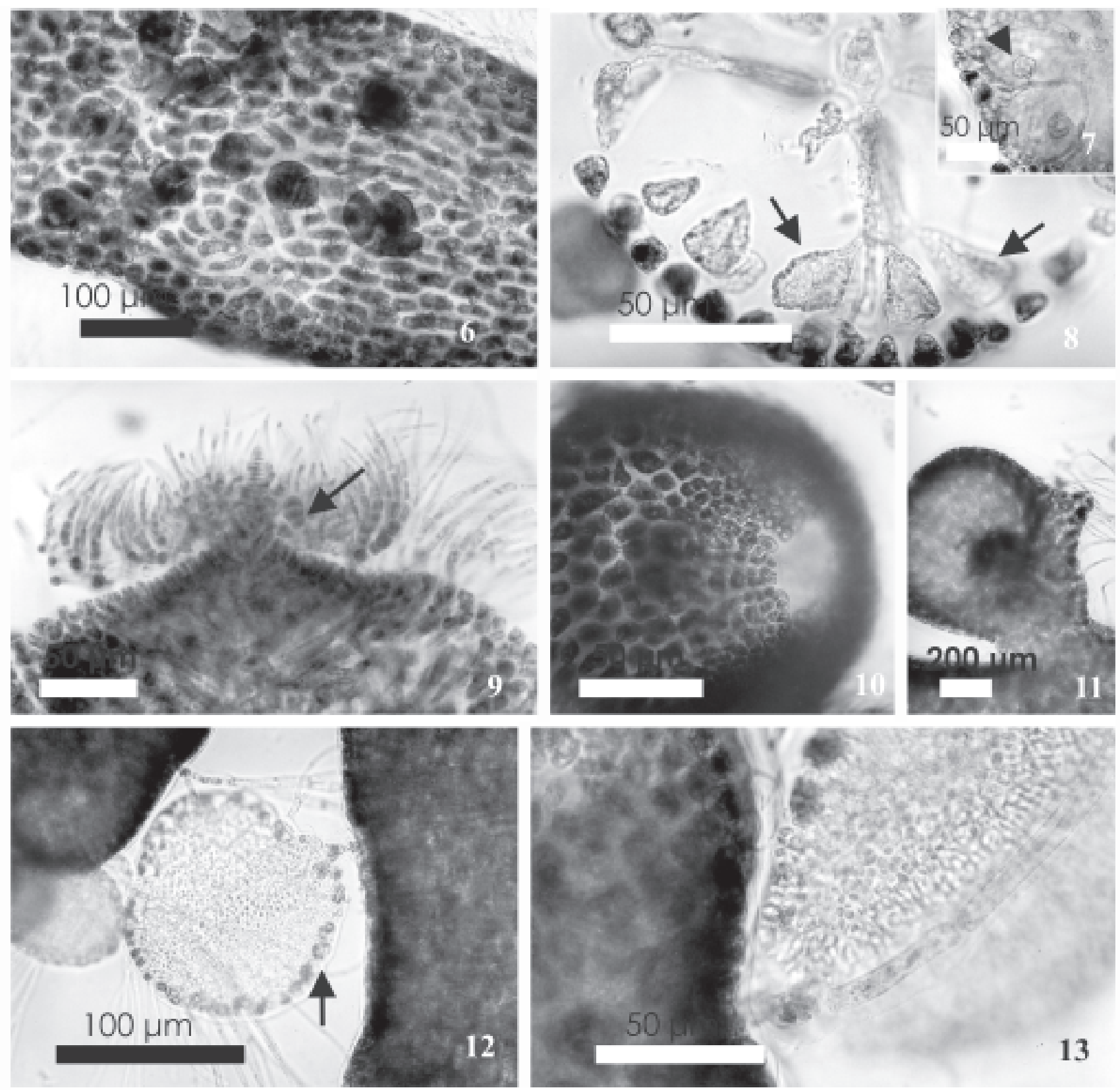

Figuras 8-12. Chondria curvilineata Collins \& Hervey. 6. Detalhe da vista superficial de planta tetrasporofitica. 7. Detalhe de corte transversal, evidenciando a origem do tetrasporângio a partir de célula pericentral (ponta de seta). 8. Corte transversal mostrando tetrasporângios sendo formados nas porções distais de células pericentrais (setas). 9. Detalhe de ápice com iniciais do ramo carpogonial. 10. Vista frontal do poro do cistocarpo. 11. Detalhe de um cistocarpo maduro. 12. Detalhe da planta com corpo espermatangial, apresentando apenas uma camada de células estéreis (seta). 13. Detalhe de um copo espermatangial. 
muito próximas às observadas neste estudo, como 40-100×10-30 $\mu \mathrm{m}$. A Tab. 1 é uma adaptação da apresentada por Tani \& Masuda (2003) e tem a finalidade de comparar as espécies morfologicamente relacionadas à espécie do presente estudo, cujas características morfológicas corroboram a presente identificação.

Embora C. minutula Weber-van Bosse, C. xishaensis Vandermeulen e $C$. simpliciuscula Weber-van Bosse estejam morfologicamente relacionadas a $C$. curvilineata por apresentarem espessamento das paredes das células medulares, essas espécies possuem características distintas das observadas na presente espécie. C. minutula apresenta somente 2 tetrasporângios por segmento axial fértil e 2-3 camadas corticais (Weber-van Bosse 1923; Cribb 1983; Price \& Scott 1992). C. xishaensis, apresenta 1 tetrasporângio por segmento e 2-3 camadas corticais (Zhang et al. 1980; Tani \& Masuda 2003). C. simpliuscula apresenta projeção de células superficiais (Weber-van Bosse 1923; Price \& Scott 1992) e esta característica não é observada nos espécimes estudados.

A presença de hápteros como na presente espécie não tem sido descrita em $C$. curvilineata (Collins \& Hervey 1917; Schneider \& Searles 1991; Tani \& Masuda 2003). Entretanto, esta diferença morfológica pode ser produzida por pressões ambientais diferenciadas, como o hidrodinamismo.

Dentre as espécies descritas para o Brasil, apenas C. leptacremon (Melvill) De Toni referida para o estado do Espírito Santo (Oliveira Filho 1969) apresenta alguma semelhança com a espécie analisada, devido a presença de eixo prostrado e ramos eretos de até $4 \mathrm{~cm}$ compr., 1-2 camadas de células corticais e presença de espessamentos lenticulares nas paredes das células medulares. Entretanto, as células corticais das camadas mais externas apresentam-se de forma irregular enquanto que as de C. curvelineata são regularmente estreitas e alongadas alternando em fileiras. Outras características diagnósticas tais como a presença de constrição proximal em ramificações e o número de células estéreis dos corpos espermatangiais não foram descritas em C. leptacremon.

Para o Estado de Santa Catarina, foi descrita C. polyrhiza por Cordeiro-Marino (1978). Entretanto, a presente espécie é facilmente separada da anterior visto que $C$. polyrhiza apresenta célula apical imersa, poucos tricoblastos emergindo do ápice e ausência de espessamentos lenticulares nas paredes das células pericentrais, além de ausência de constrições proximais dos ramos laterais. Segundo Cordeiro-Marino (1978), o arranjo das células superficiais é de circular a poliédrico, enquanto que na espécie estudada, estas são estreitas e alongadas paralelamente ao eixo principal.

Tani \& Masuda (2003) dividiram o gênero Chondria em dois grupos, um contendo espécies com ápice

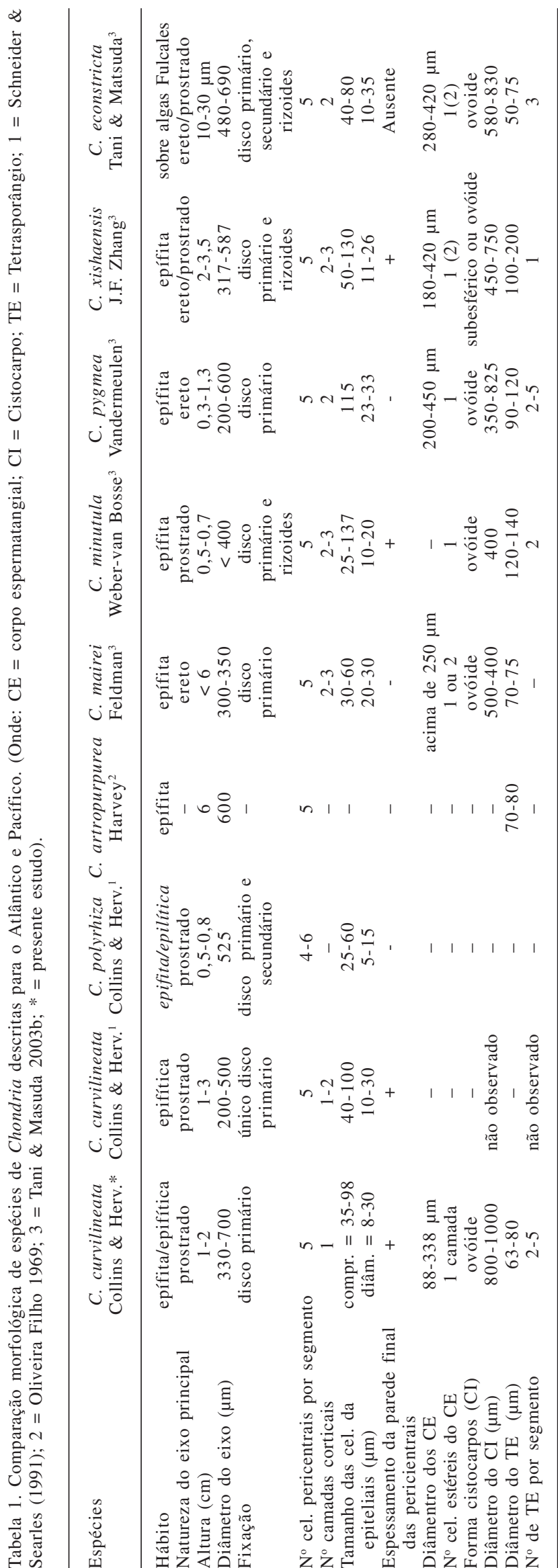


pontiagudo e outro com ápice truncado, colocando C. polyrhiza no primeiro grupo. Entretanto, Joly (1965) e Cordeiro-Marino (1978) descreveram C. polyrhiza apresentando ápice imerso em depressão, diferente do descrito por Tani \& Masuda (2003). Assim, recomendase que novas coletas sejam realizadas nas localidades listadas por Cordeiro-Marino (1978) para um posicionamento taxonômico adequado dos referidos espécimes. Esta é a primeira citação de $C$. curvelineata para o litoral brasileiro.

\section{Agradecimentos}

À CAPES, pela bolsa de estudos concedida à primeira autora.

\section{Referências bibliográficas}

Audiffred, P.A.J. \& Weisscher, F.L.M. 1984. Marine algae of Selvagem Grande (Salvage Islands, Macaronesia). Boletim do Museu Municipal do Funchal 36: 5-37.

Bacci, D.S. 2005. Estudos taxonômicos do gênero Chondria (Ceramiales, Rhodophyta) no litoral dos estados de São Paulo e Espírito Santo, Brasil. Dissertação de Mestrado. São Paulo, Instituto de Botânica.

Cabrera, R.; Moreira, A. \& Suárez, A.M. 2004. Variación en la composición y estructura de las asociaciones algales en la Bahía de Nuevitas, Costa NE de Cuba. Revista de Investigaciones Marinas 25: 133-142

Collins, F.S. \& Hervey, A.B. 1917. The Algae of Bermuda. Proceedings of the American Academy of Arts and Sciences 53: 1-195.

Cordeiro-Marino, M. 1978. Rodofíceas Marinhas do Estado de Santa Catarina. Rickia 7: 1-243.

Cormaci, M.; Furnari, G.; Giaccone, G. \& Serio, D. 2004. Alien macrophytes in the Mediterranean Sea: a review. Recent Research Developments in Environmental Biology 1: 153-202

Cribb, A.B. 1983. Marine algae of the southern Great Barrier Reef. Part I. Rhodophyta. Brisbane, Australian Coral Reef Society.

Gómez Garreta, A.; Gallardo, T.; Ribera, M. A.; Cormaci, M.; Furnari, G.; Giaccone, G. \& Boudouresque, C.-F. 2001. Checklist of the Mediterranean seaweeds. III. Rhodophyceae. 1. Ceramiales. Botanica Marina 44: 425-460

John, D.M.; Prud'homme van Reine, W.F.; Lawson, G.W.; Kostermans, T.B. \& Price, J.H. 2004. A taxonomic and geographical catalogue of the seaweeds of the western coast of Africa and adjacent Islands. Nova Hedwigia 127: 1-339.

Joly, A.B. 1957. Contribuição ao conhecimento da flora ficológica marinha da Baía de Santos e arredores. Boletim da Faculdade de Filosofia e Ciências da Universidade de São Paulo 14: $1-196$.

Joly, A.B. 1965. Flora Marinha do Litoral do Norte do estado de São Paulo e Regiões circunvizinhas. Boletim da Faculdade de Filosofia e Ciências. Universidade de São Paulo 21: 1-393.
Lipkin, Y. \& Silva, P.C. 2002. Marine algae and seagrasses of the Dahlak Archipelago, southern Red Sea. Nova Hedwigia 75: 1-90.

Littler, D.S. \& Littler, M.M. 2000. Caribbean reef plants. An identification guide to the reef plants of the Caribbean, Bahamas, Florida and Gulf of Mexico. Washington, OffShore Graphics.

Meñez, E.G. \& Calumpong, H.P. 1981. Phycological results of the 1978-1979 Smithsonian Institution Philippines Expeditions of 1978-1979 to Central Visayas, Philippines. Proceedings of the $4^{\text {th }}$ International Coral Reef Symposium 2: 379-384.

Oliveira Filho, E.C. 1969. Algas marinhas do sul do Estado do Espírito Santo (Brasil). I. Ceramiales. Boletim da Faculdade de Filosofia, Ciências e Letras da Universidade de São Paulo (Série Botânica)26: 1-277.

Price, J.H.; John, D.M. \& Lawson, G.W. 1986. Seaweeds of the western coast of tropical Africa and adjacent islands: a critical assessment. III. Rhodophyta (Florideae). 1. Genera A-F. Bulletin of the British Museum (Natural History) Botany 15: $1-122$.

Price, I.R. \& Scott, F.J. 1992. The turf algal flora of the Great Barrier Reef. Part I. Rhodophyta. Townsville, James Cook University of North Queensland.

Schneider, C.W. \& Searles, R.B. 1991. Seaweeds of the Southeastern United States. Cape Hatteras to Cape Canaveral. Durham, Duke University Press.

Silva, P.C.; Basson, P.W. \& Moe, R.L. 1996. Catalogue of the benthic marine algae of the Indian Ocean. University of California Publications in Botany 79: 1-1259.

Silva, P.C.; Meñez, E.G. \& Moe, R.L. 1987. Catalogue of the benthic marine algae of Philippines. Smithsonian Contributions to the Marine Sciences 27: 1-179.

Tani, M. \& Masuda, M. 2003. A Taxonomic study of two minute species of Chondria (Ceramiales, Rhodophyta) from the northwestern Pacific, with the desciption of Chondria econstricta sp. nov. Phycologia 4: 21-30.

Tani, M.; Yamagishi, Y.; Masuda, M.; KogameK, K.; Kawaguchi S. \& Phang, S. M. 2003. Taxonomic notes on marine algae from Malaysia. IX. Four species of Rhodophyceae, with the description of Chondria decidua sp. nov. Botanica Marina 46: $24-35$.

Taylor, W.R. 1960. Marine Algae on the Eastern Tropical and Subtropical Coasts of the Americas. Michigan, University of Michigan Press.

Ugadim, Y. 1976. Ceramiales (Rhodophyta) do litoral sul do Estado de São Paulo e do litoral do Estado do Paraná (Brasil). Boletim de Botânica da Universidade de São Paulo 4: 133-173.

Weber-van Bosse, A. 1923. Liste des algues du Siboga. III. Rhodophyceae. Seconde partie. Ceramiales. Siboga-Expedite Monographie 59c: 311-392.

Wiseman, D.R. 1976. Observations of the vegetative morphology of the red algal genus Octhodes J. Agardh (Rhizophyllidaceae, Gigartinales). Phycologia 15: 143-147.

Wynne, M.J. 1993. Benthic marine algae from the Maldives, Indian Ocean, collected during the R/V Te Vega Expedtion. Contributions from the University of Michigan Herbarium 19: 5-30.

Zhang, J.F.; Chang, C.F. \& Xia, B.M. 1980. Studies on some red algae of the Xisha Islands, Guangdong Province, China. III. Studia Marina Sinica 17: 49-68. 